\title{
Stability, Instability and Complex Behavior in Macrodynamic Models with Policy Lag
}

\author{
TOICHIRO ASADA ${ }^{\mathrm{a}, *}$ and HIROYUKI YOSHIDA ${ }^{\mathrm{b}, \dagger}$ \\ ${ }^{\mathrm{a}}$ Faculty of Economics, Chuo University 742-1 Higashinakano, Hachioji, Tokyo 192-0393, Japan; \\ ${ }^{\mathrm{b}}$ Faculty of Economics, Nagoya Gakuin University 1350 Kamishinanocho, Seto, Aichi 480-1298, Japan
}

(Received 6 June 2000)

\begin{abstract}
We construct simple macrodynamic models with policy lag by means of mixed difference and differential equations, and study how lags in policy response affect the macroeconomic (in)stability. Local dynamics of the prototype model are studied analytically, and the global dynamics of the prototype and the extended models are studied by means of numerical simulations. We show that the government can stabilize the intrinsically unstable economy if the policy lag is sufficiently short, but the system becomes locally unstable when the policy lag is too long. We also show the existence of cycles and complex behavior in some range of the policy lag.
\end{abstract}

Keywords: Policy lag; Mixed difference and differential equations; Dynamic stability; Hopf bifurcation; Complex behavior

\section{INTRODUCTION}

In a classical paper, Friedman (1948) expressed the view that the government's stabilization policy may be in fact destabilizing because of the existence of the lags in policy response. However, his argument is rather intuitive and his conclusion is not derived analytically from the formal model of macroeconomic interdependency. Without doubt, the analysis of policy lag is important from the practical as well as theoretical point of view. Nevertheless, even now there exist only a few formal analyses of policy lag. ${ }^{1}$ In this paper, we construct simple macrodynamic models with policy lag and study how lags in policy response affect the macroeconomic (in)stability. In the next section, we formulate formal models with policy lag by means of nonlinear mixed difference and differential equations (delay differential equations). Prototype model is reduced to the system with only one variable, real national income $(Y)$. Extended version of the model is expressed by the system with two variables, real national income $(Y)$ and real capital stock $(K)$. In section three, we

*Corresponding author. e-mail: asada@tamacc.chuo-u.ac.jp

†e-mail: hyoshida@ngu.ac.jp

${ }^{1}$ Phillips (1957); Asada (1991) and Yoshida (1999) are examples of such works. 
study the local dynamics of the prototype model analytically, and the conditions for local stability, local instability, and cyclical movement around the equilibrium point are detected by means of the linearization method. In section four, we study the global dynamics of prototype and extended models by using the numerical simulations. We show that the government can stabilize the intrinsically unstable economy if the policy lag is sufficiently short, but the system becomes locally unstable when the policy lag is too long. We also show the existence of cycles and complex behavior in some range of the policy lag and other parameters.

\section{THE MODEL}

Basic system of equations in our model is expressed as follows. ${ }^{2}$

$$
\begin{gathered}
\dot{Y}(t)=\alpha[C(t)+I(t)+G(t)-Y(t)] ; \quad \alpha>0 \\
C(t)=c(Y(t)-T(t))+C_{0} ; \quad 0<c<1, \quad C_{0} \geqq 0 \\
I(t)=I(Y(t), K(t), r(t)) ; I_{Y} \equiv \partial I / \partial Y>0, \\
I_{k} \equiv \partial I / \partial K<0, \quad I_{r} \equiv \partial I / \partial r<0 \\
T(t)=\tau Y(t)-T_{0} ; \quad 0<\tau<1, \quad T_{0} \geqq 0 \\
M / p=L(Y(t), r(t)) ; \quad L_{Y} \equiv \partial L / \partial Y>0 \\
L_{r} \equiv \partial L / \partial r<0 \\
M / p=\text { const. }>0 \\
G(t)=G_{0}+f(Y(t-\theta)) ; \quad f^{\prime}(Y(t-\theta)) \leqq 0 ;
\end{gathered}
$$

where $Y=$ real national income, $C=$ real private consumption expenditure, $I=$ real private investment expenditure, $G=$ real government expenditure, $T=$ real income tax, $K=$ real capital stock, $r=$ nominal rate of interest, $M=$ nominal money supply, $p=$ price level, $t=$ time period, $\theta=$ policy lag.

Equation (1) is the quantity adjustment process in the goods market. This equation implies that the real output fluctuates according as the excess demand in the goods market is positive or negative. Equations (2) through (5) are consumption function, investment function, income tax function, and equilibrium condition for money market respectively. Eq. (6) implies that the real money supply $(M / p)$ is fixed, which is merely a simplifying assumption. Eq. (7) is the government's policy function with the delay in policy response to national income.

Solving Eq. (5) with respect to $r$, we have the following 'LM equation'.

$$
r(t)=r(Y(t)) ; \quad r_{Y} \equiv r^{\prime}(Y)=-L_{Y} / L_{r}>0
$$

Substituting Eq. (4) into Eq. (2), and substituting Eq. (8) into Eq. (3), we obtain the following expressions.

$$
\begin{gathered}
C(t)=c(1-\tau) Y(t)+C_{0}+c T_{0} \\
I(t)=I(Y(t), K(t), r(Y(t)))
\end{gathered}
$$

Substituting Eqs. (7), (9), and (10) into Eq. (1), we have

$$
\begin{aligned}
\dot{Y}(t)=\alpha[ & I(Y(t), K(t), r(Y(t))) \\
& -\{1-c(1-\tau)\} Y(t)+C_{0} \\
& \left.+c T_{0}+G_{0}+f(Y(t-\theta))\right] .
\end{aligned}
$$

This is single dynamical equation with two endogenous variables $(Y, K)$. Therefore, this system is not yet complete. We need one more equation to close the model. In this paper, we shall consider two ways to close the model.

First, let us consider the 'short run' model in the sense that the real capital stock is fixed, i.e.,

$$
K=\bar{K} .
$$

\footnotetext{
${ }^{2}$ This formulation is essentially based on Asada (1991), and it is the dynamic version of rather standard Keynesian macroeconomic model.
} 
Substituting Eq. (12) into Eq. (11), we obtain the following prototype model.

$$
\begin{array}{rl}
\dot{Y}(t)=\alpha[ & I(Y(t), \bar{K}, r(Y(t))) \\
& -\{1-c(1-\tau)\} Y(t) \\
& \left.+C_{0}+c T_{0}+G_{0}+f(Y(t-\theta))\right] \\
\equiv F & F(Y(t), Y(t-\theta))
\end{array}
$$

which is a simple type of mixed difference and differential equation (delay differential equation). ${ }^{3}$ We shall call the model which is summarized in the system $\left(S_{1}\right)$ 'model 1'.

An extended version of our model is the 'intermediate run' model in which the capital stock becomes an endogenous variable. In this case, we allow for the fact that the investment contributes to change the level of capital stock, so that we replace Eq. (12) with the following equation.

$$
\dot{K}(t)=I(Y(t), K(t), r(Y(t)))
$$

This model, which we call 'model 2', is reduced to the following system of equations.

$$
\begin{aligned}
\dot{Y}(t)=\alpha[ & I(Y(t), K(t), r(Y(t))) \\
& -\{1-c(1-\tau)\} Y(t)+C_{0}+c T_{0}+G_{0} \\
& +f(Y(t-\theta))] \equiv F_{1}(Y(t), Y(t-\theta), K(t))
\end{aligned}
$$

$$
\dot{K}(t)=I(Y(t), K(t), r(Y(t))) \equiv F_{2}(Y(t), K(t)) \quad\left(S_{2}\right)
$$

'Model 2' is more akin to Kaldor (1940)'s business cycle theory than 'model 1' in spirit. We shall study 'model 1' analytically and numerically, but we shall study 'model 2' only numerically.

\section{LOCAL DYNAMICS OF 'MODEL 1': A MATHEMATICAL ANALYSIS}

In this section, we shall investigate the local dynamics of 'model 1' analytically by means of the linear approximation method.

Let us assume that there exists an equilibrium solution $Y^{*}>0$ of the system $\left(S_{1}\right)$ which satisfies

$$
\begin{gathered}
F\left(Y^{*}, Y^{*}\right)=\alpha\left[I\left(Y^{*}, \bar{K}, r\left(Y^{*}\right)\right)-\{1-c(1-\tau)\} Y^{*}\right. \\
\left.+C_{0}+c T_{0}+G_{0}+f\left(Y^{*}\right)\right]=0 .{ }^{4}
\end{gathered}
$$

Expanding the system $\left(S_{1}\right)$ in a Taylor series around the equilibrium point $Y^{*}$ and neglecting the terms of higher order than the first order, we have the following linear approximation of the system $\left(S_{1}\right)$.

$$
\dot{y}(t)=\alpha a y(t)-\alpha \beta y(t-\theta) ;
$$

where $\quad a \equiv[\partial \dot{Y}(t) / \partial Y(t)]^{*} / \alpha \equiv I_{Y}^{*}+I_{r}^{*} r_{Y}^{*}-\{1-c$ $(1-\tau)\}, \beta \equiv-[\partial \dot{Y}(t) / \partial Y(t-\theta)]^{*} / \alpha \equiv-f^{\prime}\left(Y^{*}\right) \geqq 0$, $y(t) \equiv Y(t)-Y^{*}$, and $y(t-\theta) \equiv Y(t-\theta)-Y^{*}{ }^{5}$ Now, let us assume

Assumption A1

$$
a \equiv I_{Y}^{*}+I_{r}^{*} r_{Y}^{*}-\{1-c(1-\tau)\}>0 .
$$

Assumption A1 implies that the propensity to invest $\left(I_{Y}^{*}\right)$ at the equilibrium point is considerably large, which is a basic assumption of Kaldor (1940)'s business cycle theory.

We can study the local dynamics of the system $\left(S_{1}\right)$ in the vicinity of the equilibrium point by studying the dynamics of the linearized system (15). Substituting $y(t)=y(0) e^{\rho t}$ into Eq. (15), we obtain the following 'characteristic equation'.

$$
\Gamma(\rho) \equiv \rho-\alpha a+\alpha \beta e^{-\theta \rho}=0
$$

\footnotetext{
${ }^{3}$ As for the mathematics of such an equation, see Bellman and Cooke (1963); Kuang (1993) and Gandolfo (1993) Chap. 27. There are a few examples of the applications of such an equation in economic literatures. See, among others, Kalecki (1935); Steindl (1952); Johansen (1959); Lange (1969); Mackey (1989); Asada (1991, 1994); Ioannides and Taub (1992); Asea and Zak (1999), and Yoshida (1999).

${ }^{4}$ We need not assume that $Y^{*}$ is unique. In fact, we shall present a numerical example with multiple equilibria in Section 4.

${ }^{5}$ The asterisk $\left(^{*}\right)$ shows that the values are evaluated at the equilibrium point.
} 
or equivalently,

$$
(1 / \theta) \lambda-\alpha a+\alpha \beta e^{-\lambda}=0
$$

where $\lambda \equiv \theta \rho$. If all the roots of Eq. (17) have negative real parts, the equilibrium point of the system $\left(S_{1}\right)$ is locally stable. On the other hand, it becomes locally unstable if at least one root of Eq. (17) has positive real part. ${ }^{6}$ First, let us consider the characteristics of the real roots of Eq. (17).

\subsection{Characteristics of the Real Roots ${ }^{7}$}

We can rewrite Eq. (17) as

$$
f_{1}(\lambda) \equiv e^{-\lambda}=-(1 / \theta \alpha \beta) \lambda+a / \beta \equiv f_{2}(\lambda) .
$$

We can see from Figure 1 that Eq. (17) has one positive real root and one negative real root when $0<\beta<a$.

Figure 2 illustrates the case of $\beta=a$. In this case, $\lambda=0$ is always one of the roots of Eq. (18). In addition, (i) we have one negative real root when $\theta<1 / \alpha a$ and $\beta=a$, and (ii) we have one positive real root when $\theta>1 / \alpha a$ and $\beta=a$.

The case of $\beta>a$ is illustrated in Figure 3. This figure shows that

(i) Eq. (17) has two negative real roots when $\theta$ is sufficiently small,

(ii) it has two positive real roots when $\theta$ is sufficiently large, and

(iii) it has no real root at the intermediate values of $\theta$.

Next, we shall consider the mathematical condition for the existence of the multiple real roots of Eq. (17). This condition is given by

$$
f_{1}^{\prime}(\lambda) \equiv-e^{-\lambda}=-(1 / \theta \alpha \beta) \equiv f_{2}^{\prime}(\lambda)
$$

or equivalently,

$$
e^{\lambda}=\theta \alpha \beta
$$

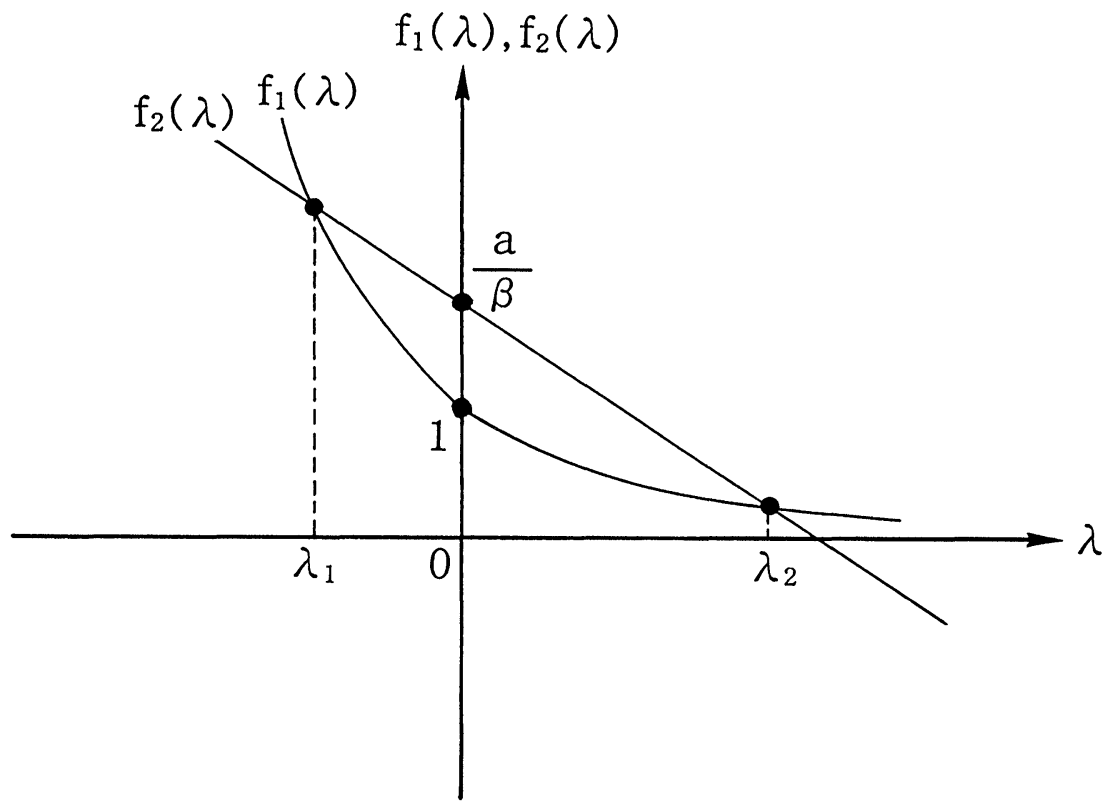

FIGURE $1 \quad(0<\beta<a)$.

\footnotetext{
${ }^{6}$ As for the proof, see, for example, Bellman and Cooke (1963) Chap. 11. As for the significance of the characteristic root approach to the mixed difference and differential equations, see Frisch and Holme (1935) and James and Belz (1938).

${ }^{7}$ Subsections 3.1 and 3.2 are essentially based on Asada (1997) Chap. 2.
} 


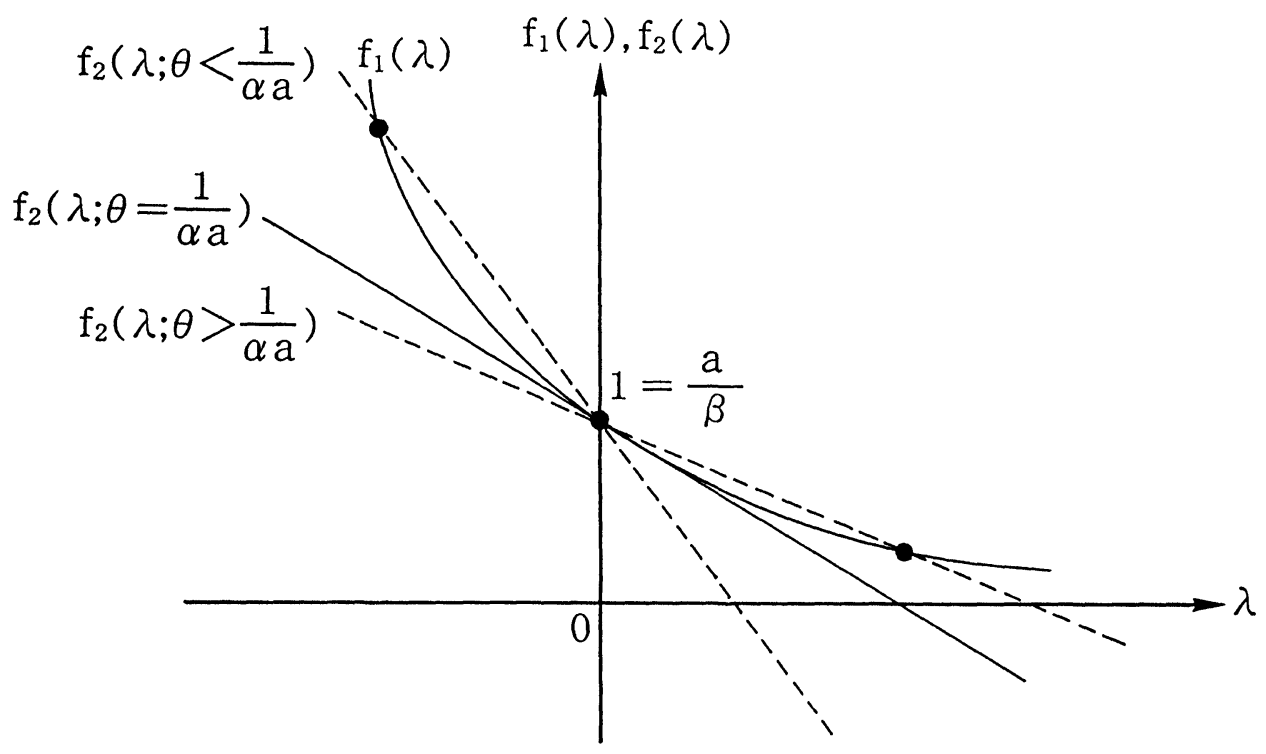

FIGURE $2(\beta=a)$.

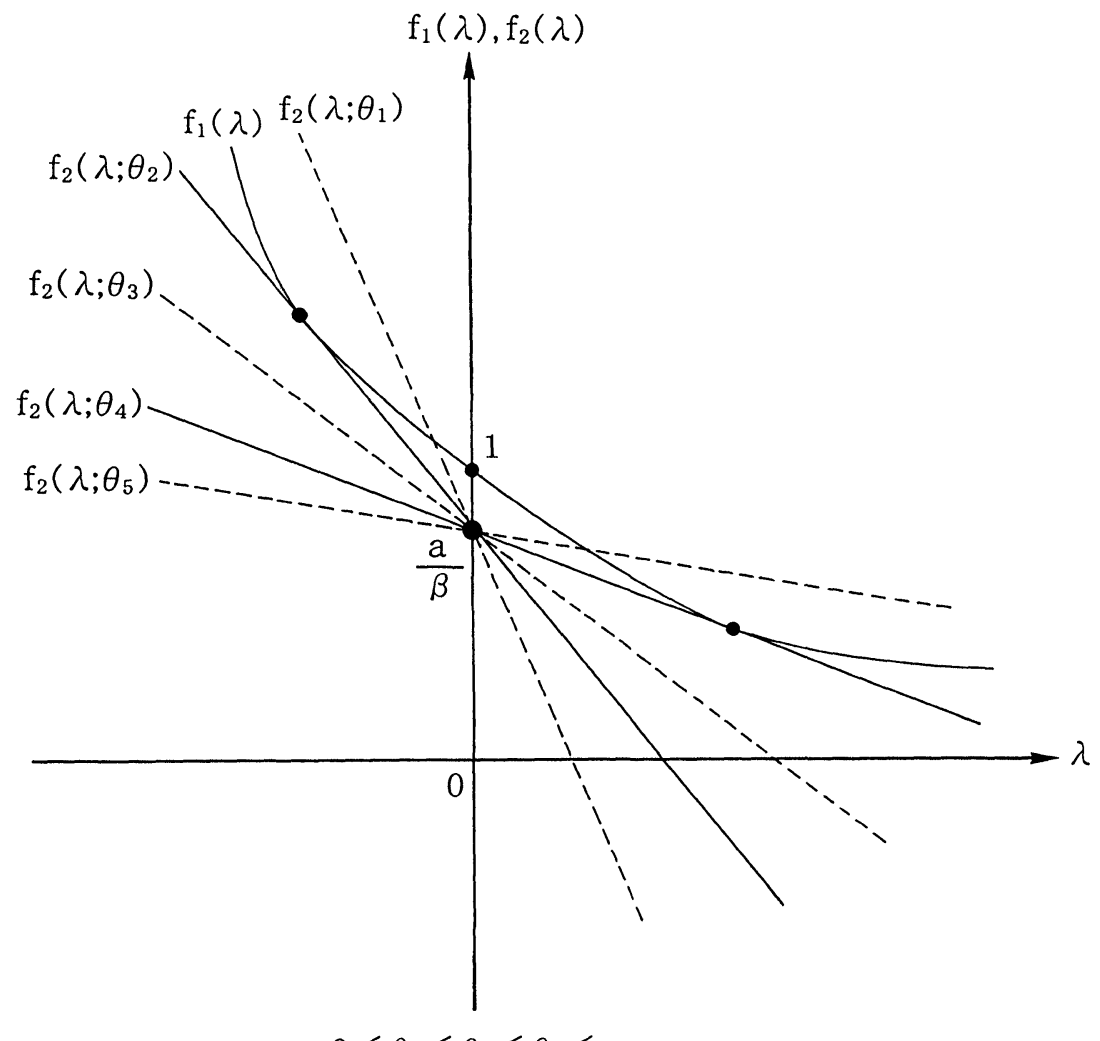

$0<\theta_{1}<\theta_{2}<\theta_{3}<\cdots$

FIGURE $3(\beta>a)$. 
This condition is also equivalent to

$$
\lambda=\log (\theta \alpha \beta) .
$$

Substituting Eqs. (20) and (21) into Eq. (18), we have

$$
(1 / \theta \alpha \beta)=-(1 / \theta \alpha \beta) \log (\theta \alpha \beta)+a / \beta
$$

or equivalently,

$$
\log (\theta \alpha \beta)=\theta \alpha a-1 .
$$

Solving Eq. (23) with respect to $\beta$, we obtain the following expression.

$$
\begin{aligned}
\beta & =(1 / \theta \alpha) e^{\theta \alpha a-1} \equiv \varphi(\theta) ; \\
\varphi^{\prime}(\theta) & =\left\{(\theta \alpha a-1) / \alpha \theta^{2}\right\} e^{\theta \alpha a-1} \\
& \geqq 0 \Leftrightarrow \theta \gtreqless 1 / \alpha a ; \\
\varphi(1 / \alpha a) & =a, \quad \lim _{\theta \rightarrow 0} \varphi(\theta)=+\infty .
\end{aligned}
$$

We can summarize the results of the above analysis as in Figure 4 and Table I.

\subsection{Local Stability/Instability Analysis}

Table I shows that the equilibrium point of the system $\left(S_{1}\right)$ is locally unstable in the region $A \cup B$. But, it is necessary to obtain the information on the complex roots to study the local stability/ instability in the region $C \cup D{ }^{8}$ For this purpose, we can utilize the following mathematical result which is due to Hayes (1950) to get full information on the local stability of the system.

Lemma 1 (Hayes' theorem) All the roots of $H(\lambda) \equiv p e^{\lambda}+q-\lambda e^{\lambda}=0$, where $p$ and $q$ are real, have negative real parts if and only if

(i) $p<1$, and

(ii) $p<-q<\sqrt{ }\left(x^{* 2}+p^{2}\right)$,

where $x^{*}$ is the root of $x=p$ tan $x$ such that $0<x<\pi$. If $p=0$, we take $x^{*}=\pi / 2$.

Proof See Hayes (1950) or Bellman and Cooke (1963) Chap. 13.

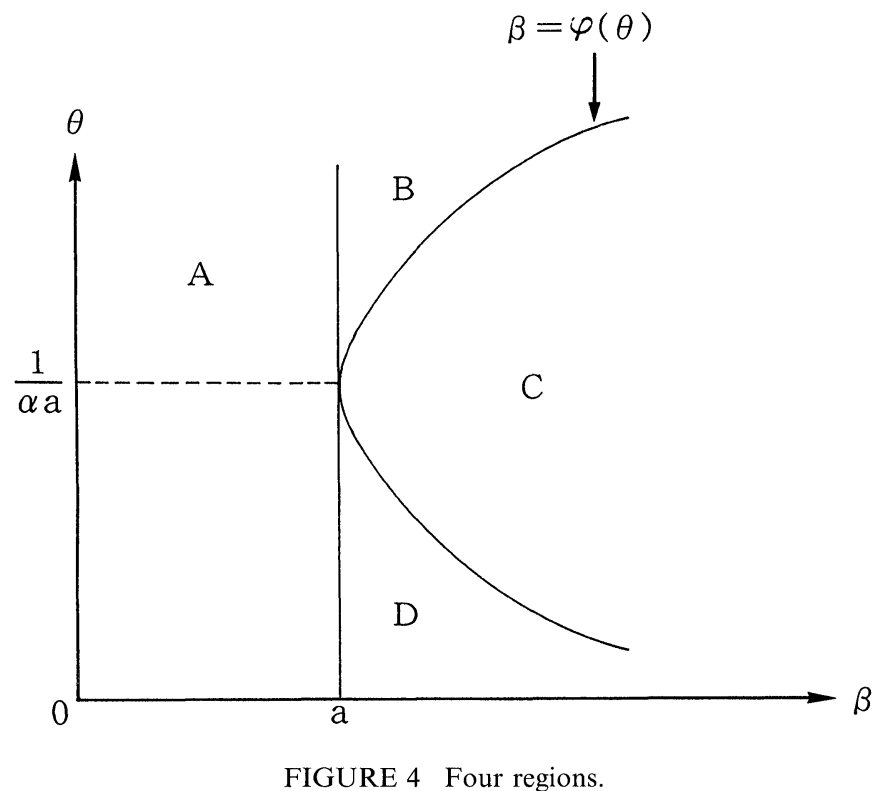

\footnotetext{
${ }^{8}$ In fact, we can show that Eq. (17) has infinite number of complex roots.
} 
TABLE I Classification of the regions

\begin{tabular}{ll}
\hline Region & $\begin{array}{c}\text { Characteristics of } \\
\text { the real roots }\end{array}$ \\
\hline A & one positive, \\
& one negative \\
B & two positive \\
C & no real root \\
D & two negative \\
\hline
\end{tabular}

We can rewrite the characteristic Eq. (17) as

$H(\lambda) \equiv \theta \alpha a e^{\lambda}+(-\theta \alpha \beta)-\lambda e^{\lambda} \equiv p e^{\lambda}+q-\lambda e^{\lambda}=0$

where $p \equiv \theta \alpha a$ and $q \equiv-\theta \alpha \beta$. It follows from Lemma 1 that we can express a set of the necessary and sufficient conditions for local stability as follows.

$$
\theta<1 / \alpha a
$$

$$
a<\beta
$$

(iii) $\beta<\sqrt{ }\left\{\left(x^{*} / \theta \alpha\right)^{2}+a^{2}\right\} \equiv \psi(\theta)$, where $x^{*}$ is the solution of $g_{1}(x) \equiv(1 / \theta \alpha a) x=$ $\tan x \equiv g_{2}(x)$ such that $0<x<\pi$.

We can illustrate the solution $x^{*}$ as in Figure 5 when the inequality (26) (i) is satisfied. ${ }^{9}$ Furthermore, we can see from Figure 5 that $\tan x^{*}$ becomes a decreasing function of $\theta$. Therefore, we have

$$
\frac{d}{d \theta}\left(x^{*} / \theta \alpha\right)=a \frac{d\left(\tan x^{*}\right)}{d \theta}<0 .
$$

We can derive the following relationships from Eq. (26) (iii) and Eq. (27). ${ }^{10}$

(i) $\psi^{\prime}(\theta)=\frac{\left(x^{*} / \theta \alpha\right)}{\sqrt{ }\left\{\left(x^{*} / \theta \alpha\right)^{2}+a^{2}\right\}} \frac{d}{d \theta}\left(x^{*} / \theta \alpha\right)<0$,

(ii) $\quad \lim _{\theta \rightarrow 0} \psi(\theta)=\lim _{\theta \rightarrow 0} a \sqrt{ }\left(\tan ^{2} x^{*}+1\right)=+\infty$

(iii) $\lim _{\theta \rightarrow 1 / \alpha a} \psi(\theta)=\lim _{\theta \rightarrow 1 / \alpha a} a \sqrt{ }\left(\tan ^{2} x^{*}+1\right)=a$.

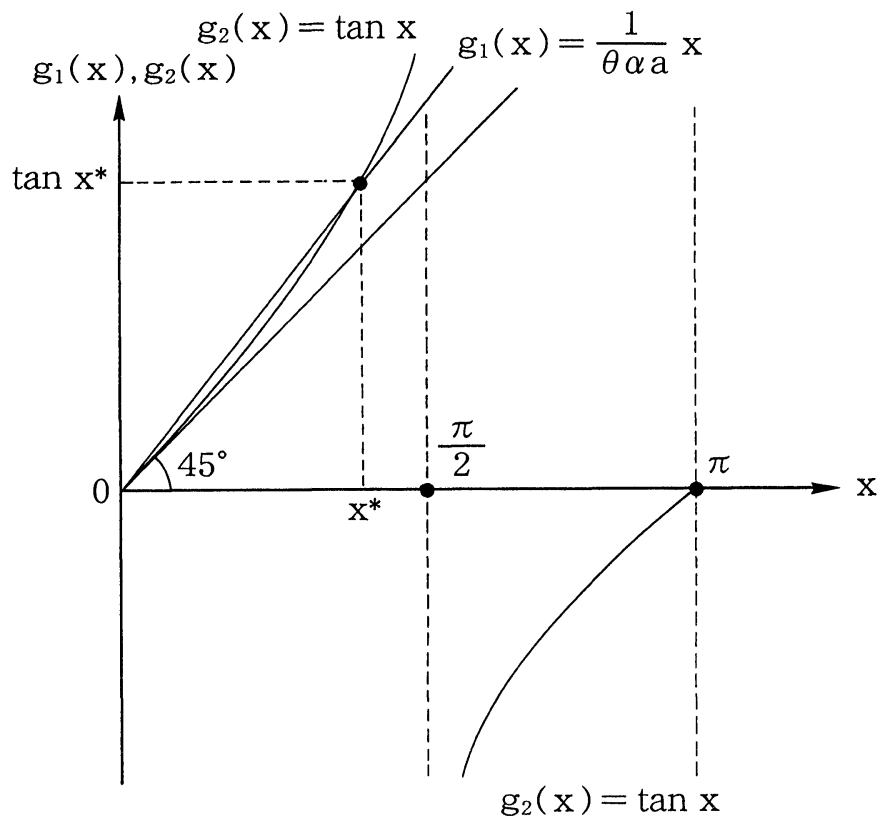

FIGURE 5 Solution of $g_{1}(x)=g_{2}(x)$.

\footnotetext{
${ }^{9}$ Note that $d(\tan x) / d x=1+\tan ^{2} 0=1$ when $x=0$, and the inequality (26)(i) implies that $1 / \theta \alpha a>1$.

${ }^{10}$ We can see from Figure 5 that $\lim _{\theta \rightarrow 0} \tan x^{*}=+\infty$ and $\lim _{\theta \rightarrow 1 / \alpha a} \tan x^{*}=0$.
} 
Now, let us define the 'stable region' $S$ as

$S \equiv\left\{(\beta, \theta) \in R_{++}^{2} \mid\right.$ All the roots of Eq. (17)

have negative real parts\}

$$
\equiv\left\{(\beta, \theta) \in R_{++}^{2} \mid \theta<1 / \alpha a, \quad a<\beta<\psi(\theta)\right\}
$$

We can express the region $S$ as in Figure 6 (boundary points are excluded). We can summarize the result of the above analysis as the following proposition.

\section{PROPOSITION 1}

(i) If $\theta>1 / \alpha a$, the equilibrium point of the system $\left(S_{1}\right)$ becomes locally unstable irrespective of the value of $\beta \geqq 0$.

(ii) If $0<\theta<1 / \alpha a$, the equilibrium point of the system $\left(S_{1}\right)$ is locally stable for $\beta \in(a, \psi(\theta))$ and it is locally unstable for $\beta \in[0, a) \cup(\psi(\theta),+\infty)$, where $\psi(\theta)$ is a continuous decreasing function of $\theta$ and $\lim _{\theta \rightarrow 0} \psi(\theta)=+\infty$ and $\lim _{\theta \rightarrow 1 / \alpha a} \psi(\theta)=a$.

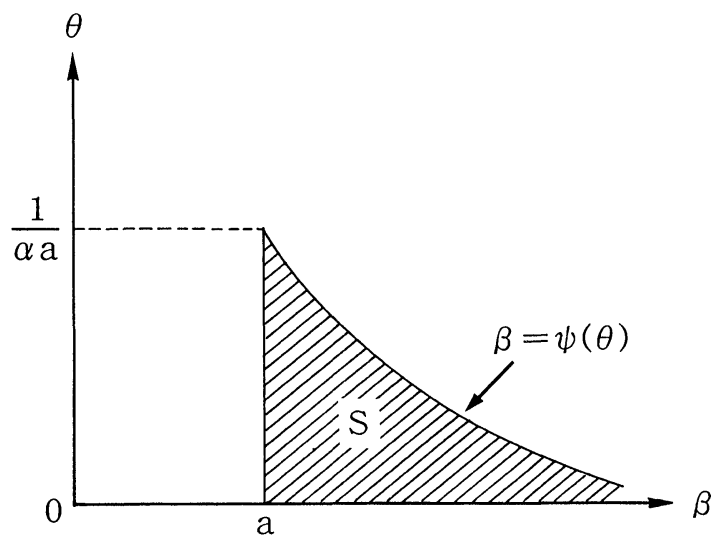

FIGURE 6 Stable region.

\subsection{Hopf-bifurcation and the Existence of the Closed Orbits}

Proposition 1 says that (i) too long delay in policy response must fail to stabilize the economy, (ii) too strong policy as well as too weak policy is unsuccessful to stabilize the economy even if the policy lag $(\theta)$ is relatively short, and (iii) the stabilization policy is successful at the intermediate range of the strength of the policy response $(\beta)$ if $\theta$ is relatively short. These analytical results seem to suggest that the pure cyclical movements will occur at the intermediate values of $\beta$ when $\theta$ is not too large. ${ }^{11}$ Now, we shall prove mathematically that this conjecture is in fact correct. We can make use of the following version of the Hopf-bifurcation theorem. ${ }^{12}$

Lemma 2 Let $\dot{x}(t)=F(x(t), x(t-\theta) ; \varepsilon), x \in R$, $\varepsilon \in R$ be a mixed difference and differential equation with a parameter $\varepsilon$. Suppose that the following properties are satisfied.

(i) This equation has smooth curve of equilibria $F\left(x^{*}, x^{*} ; \varepsilon\right)=0$.

(ii) The characteristic equation $\Gamma(\rho) \equiv \rho-a-$ $b e^{-\theta \rho}=0$ has a pair of pure imaginary roots $\rho\left(\varepsilon_{0}\right), \bar{\rho}\left(\varepsilon_{0}\right)$ and no other root with zero real part, where $a \equiv(\partial F / \partial x(t))^{*}$ and $b \equiv(\partial F /$ $\partial x(t-\theta))^{*}$ are partial derivatives of $F$ which are evaluated at $\left(x^{*}\left(\varepsilon_{0}\right), \varepsilon_{0}\right)$ with the parameter $\varepsilon_{0}$.

(iii) $d\{\operatorname{Re} \rho(\varepsilon)\} / d \varepsilon \neq 0$ at $\varepsilon=\varepsilon_{0}$, where $\operatorname{Re} \rho(\varepsilon)$ is the real part of $\rho(\varepsilon)$.

Then, there exists a continuous function $\varepsilon(\gamma)$ with $\varepsilon(0)=\varepsilon_{0}$, and for all sufficiently small values of $\gamma \neq 0$ there exists a continuous family of nonconstant periodic solutions $x(t, \gamma)$ for the above dynamical equation, which collapses to the equilibrium point $x^{*}\left(\varepsilon_{0}\right)$ as $\gamma \rightarrow 0$. The period of the cycle

\footnotetext{
${ }^{11}$ This phenomenon may be called the 'policy cycle'.

${ }^{12}$ Usually the Hopf-bifurcation theorem is applied to the system of differential equations ( $c f$. Gandolfo (1996) Chap. 25 and Asada (1997) Chap. 3). However, this theorem is also applicable to the mixed difference and differential equation (cf. Rustichini (1989) and Kuang (1993) Chap. 2). Mackey (1989) and Asea and Zak (1999) are examples of the application of the Hopf-bifurcation theorem to the mixed difference and differential equation in economic theory.
} 
is close to $2 \pi / \operatorname{Im} \rho\left(\varepsilon_{0}\right)$, where $\operatorname{Im} \rho\left(\varepsilon_{0}\right)$ is the imaginary part of $\rho\left(\varepsilon_{0}\right)$.

Now, it is clear from the analyses in Sections 3.1 and 3.2 that there exists the value $\hat{\theta} \in[0,1 / \alpha a)$ such that the following property $(P)$ is satisfied (see Fig. 7).

$(P)$ For all $\theta \in(\hat{\theta}, 1 / \alpha a)$,

$$
Z \equiv\left\{(\beta, \theta) \in R_{++}^{2} \mid \beta=\psi(\theta)\right\} \subset C
$$

where $C$ is given by Figure 4, i.e.,

$$
C \equiv\left\{(\beta, \theta) \in R_{++}^{2} \mid \beta>\varphi(\theta)\right\} .^{13}
$$

Proposition 2 Let us fix the parameter $\theta_{0} \in(\hat{\theta}, 1 / \alpha a)$ and select $\beta$ as a bifurcation parameter. Then, at $\beta_{0}=\psi\left(\theta_{0}\right)$ the Hopf-bifurcation occurs. In other words, at $\beta_{0}$, the characteristic Eq. (16) has a pair of pure imaginary roots $\rho\left(\beta_{0}\right)=$ $z_{0} i, \bar{\rho}\left(\beta_{0}\right)=-z_{0} i\left(i \equiv \sqrt{-1}, z_{0} \neq 0\right)$ and no other root with zero real part, and $d\{\operatorname{Re} \rho(\beta)\} \mid d \beta>0$ at $\beta=\beta_{0}$. Furthermore, $z_{0}<\pi / \theta_{0}$ so that we have $2 \pi / \operatorname{Im} \rho\left(\beta_{0}\right)=2 \pi / z_{0}>2 \theta_{0}$.
Proof See Appendix.

Corollary of Proposition 2 There exist some non-constant periodic solutions of the system $\left(S_{1}\right)$ at some parameter values $\beta>0$ which are sufficiently close to $\beta_{0}=\psi\left(\theta_{0}\right)$. The period of the cycle is close to $2 \pi / z_{0}>2 \theta_{0}$.

Proof It directly follows from Lemma 2 and Proposition 2.

\section{NUMERICAL SIMULATIONS}

In the previous section, we presented some analytical results on the local dynamics of 'model 1 ' around the equilibrium point. However, we must resort to the study of the numerical simulations to get some information on the global dynamics of the system. Furthermore, it is difficult to get even the information on the local dynamics by means of analytical approach if we consider more complicated system such as 'model 2'. In this section, we shall present some results of the global dynamics of 'model 1' and 'model 2' by means of numerical simulations.

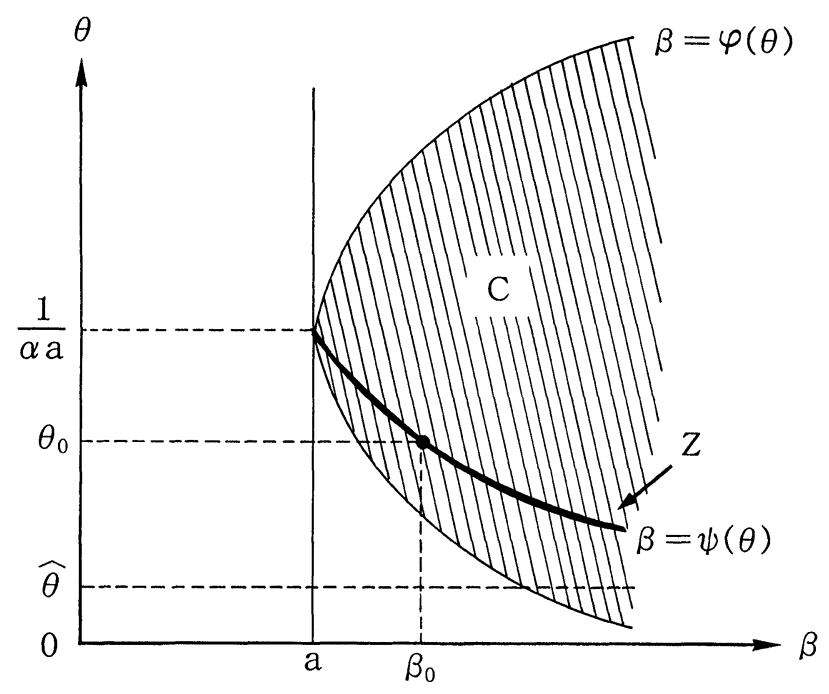

FIGURE 7 Hopf-bifurcation curve (Z).

\footnotetext{
${ }^{13}$ The functions $\psi(\theta)$ and $\varphi(\theta)$ are given in Eq. (26)(iii) and (24) respectively.
} 


\subsection{Simulation of 'Model 1'}

First, let us study 'model 1' by adopting the following specifications of the functional forms and the parameter values.

$$
\begin{gathered}
\dot{Y}(t)=\alpha[I(Y(t), \bar{K}, r(Y(t))) \\
\quad-\{1-c(1-\tau)\} Y(t)+C_{0} \\
\left.\quad+c T_{0}+G_{0}+\beta(400-Y(t-\theta))\right] \\
\equiv F(Y(t), Y(t-\theta)) \\
I(Y(t), \bar{K}, r(Y(t))) \\
=\frac{400}{1+9 \exp [-0.1(Y(t)-400)]}-0.01 \sqrt{Y(t)} \\
+0.2-40 \\
c(1-\tau)=0.5, C_{0}+c T_{0}+G_{0}=200, \alpha=0.9
\end{gathered}
$$

Eq. (33) is an example of the Kaldorian S-shaped investment function.

Figure 8 is the phase diagram of this system in $(Y(t), Y(t+0.3))$ plane in the case of $\beta=6.6$ and $\theta=0.2{ }^{14}$ Because of the S-shaped investment function, there exist three equilibrium points and three limit cycles. One equilibrium point
$\left(Y^{*}=400\right)$ is unstable and two equilibrium points $\left(Y^{* *}<400, \quad Y^{* * *}>400\right)$ are locally stable. One (large) limit cycle is stable and two (small) limit cycles are unstable. Figure 9(a) and (b) are the bifurcation diagrams of this system with respect to the parameter $\beta$ corresponding to the initial conditions $Y(0)=420$ and $Y(0)=390$ respectively. These figures show that we have different bifurcation diagrams corresponding to different initial conditions because of the multiple equilibria. ${ }^{15}$ In other words, this system has pathdependent characteristics. Figure 10 is the bifurcation diagram with respect to the policy lag $(\theta)$ when $\beta$ is fixed at $\beta=5.6$.

\subsection{Simulation of 'Model 2'}

Next, we shall consider the numerical study of 'model 2' by using the following data.

$$
\begin{aligned}
\dot{Y}(t)=\alpha[ & I(Y(t), K(t), r(Y(t))) \\
& -\{1-c(1-\tau)\} Y(t)+C_{0}+c T_{0}+G_{0} \\
& +\beta(400-Y(t-\theta))] \\
\equiv F_{1}(Y(t), Y(t-\theta), K(t)) & \\
\dot{K}(t)= & I(Y(t), K(t), r(Y(t))) \equiv F_{2}(Y(t), K(t))
\end{aligned}
$$

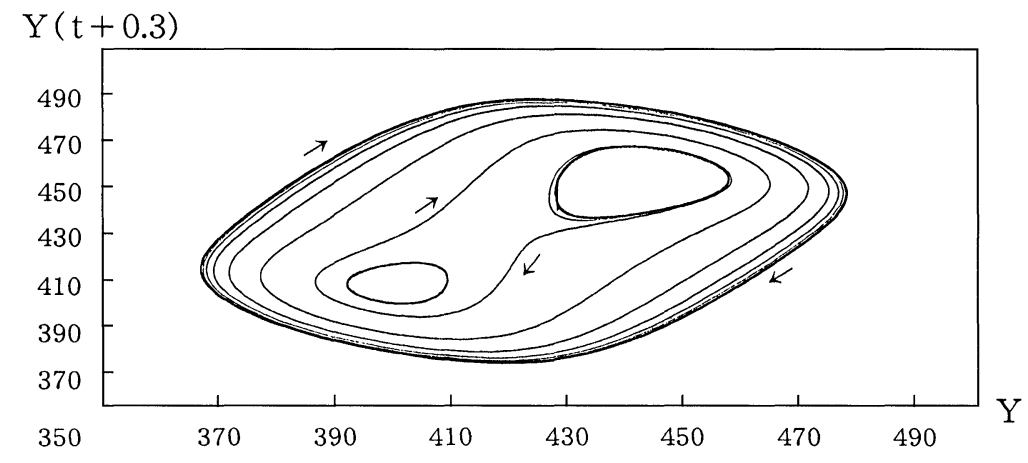

FIGURE 8 One stable limit cycle and two unstable limit cycles $(\beta=6.6, \theta=0.2)$.

\footnotetext{
${ }^{14}$ We adopted the approximation of Eq. (32) by means of 'Euler's algorithm', i.e., $Y(t+\Delta t)=Y(t)+(\Delta t) F(Y(t), \quad Y(t-\theta))$, $\Delta t=0.01$.

${ }^{15}$ In Figure 9, $\theta$ is fixed at $\theta=0.2$ and only the maximum and minimum values of $Y(t)$ are plotted.
} 

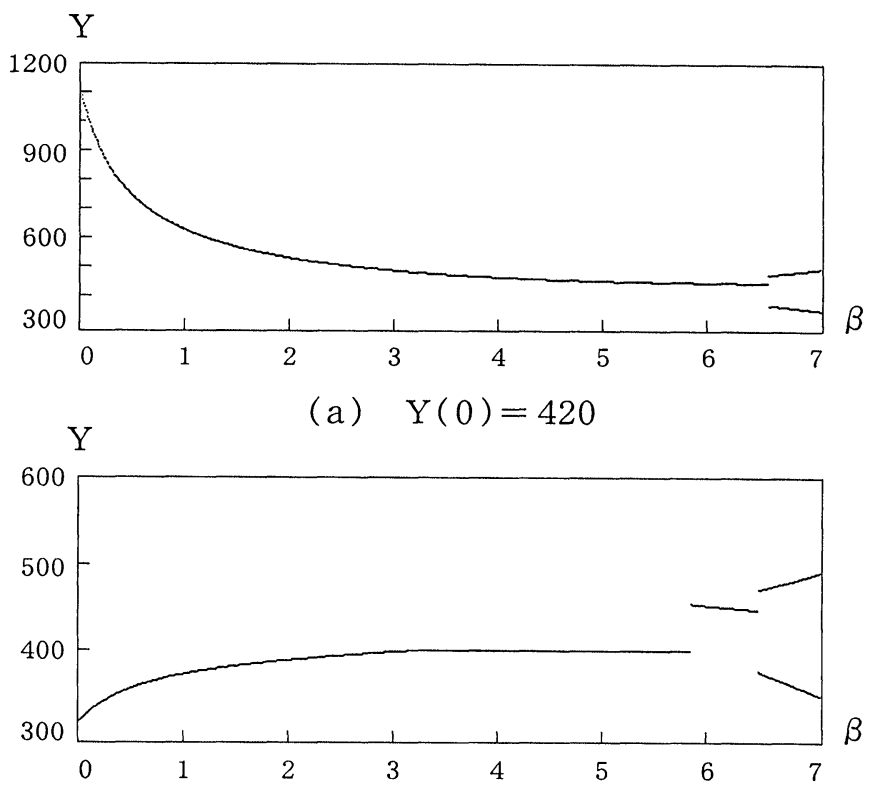

(b) $\quad \mathrm{Y}(0)=390$

FIGURE 9 Bifurcation diagrams of $Y$ when $\theta=0.2$ (parameter: $\beta$ ).

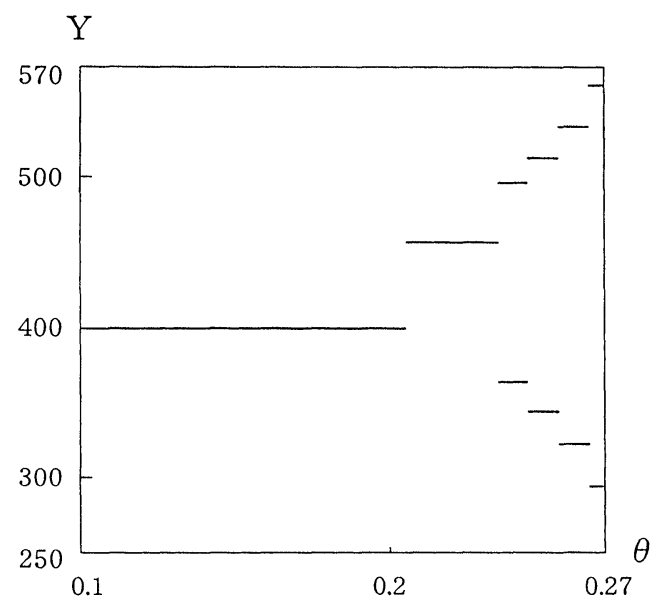

FIGURE 10 Bifurcation diagrams of $Y$ when $\beta=5.6$ (parameter: $\theta$ ).

$$
\begin{aligned}
I(Y(t), & K(t), r(Y(t))) \\
= & \frac{400}{1+12 \exp [-0.1(Y(t)-400)]} \\
& -0.01 \sqrt{Y(t)}-0.5 K(t)
\end{aligned}
$$

$$
c(1-\tau)=0.5, C_{0}+c T_{0}+G_{0}=200, \alpha=0.9
$$

Figure 11 shows that behavior of this system can be chaotic for some parameter values. This figure illustrates a strange attractor which is produced when $\beta=4.1$ and $\theta=0.3$. $^{16}$ Figure 12(a) is the bifurcation diagram with respect to the parameter $\beta$ when $\theta$ is fixed at $\theta=0.3$. Figure $12(\mathrm{~b})$ is the same bifurcation diagram at the interval $4.0 \leqq \beta \leqq 4.2$. These figures show that the limit cycles are produced for both of sufficiently small values and sufficiently large values of $\beta$. At the intermediate values of $\beta$, the equilibrium point becomes stable, and at the vicinity of the parameter value $\beta=4.1$, the behavior of the system becomes complex. Finally, Figure 13 is the bifurcation diagram with respect to the policy lag $(\theta)$ when $\beta$ is fixed at $\beta=4.1$.

\footnotetext{
${ }^{16}$ Also in this case, we adopted Euler's algorithm for the approximation of equations (35) and (36), i.e., $Y(t+\Delta t)=Y(t)+(\Delta t) F_{1}(Y(t), Y(t-\theta), K(t)), K(t+\Delta t)=K(t)+(\Delta t) F_{2}(Y(t), K(t)), \Delta t=0.01$. Contrary to the case of 'model 1', this system has only one equilibrium point $\left(Y^{*}, K^{*}\right)=(400,61.1)$.
} 


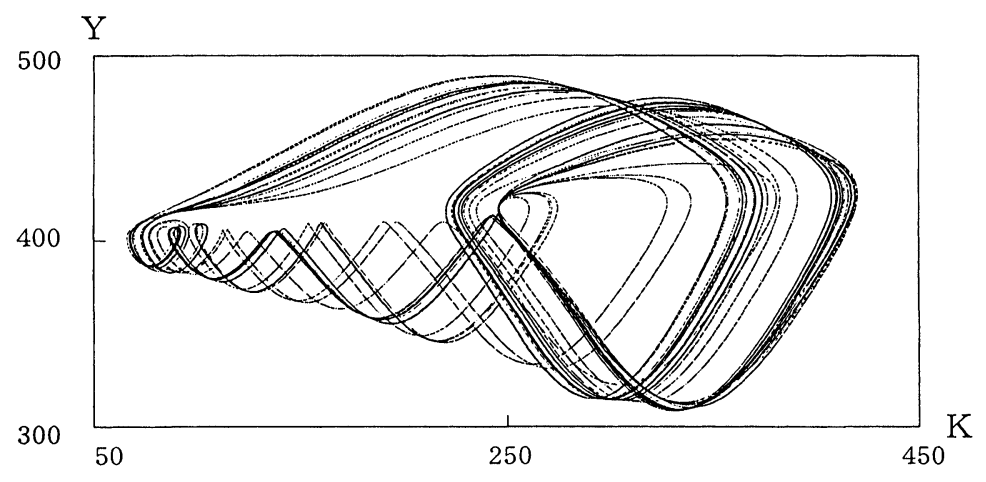

FIGURE 11 Strange attractor $(\beta=4.1, \theta=0.3)$.

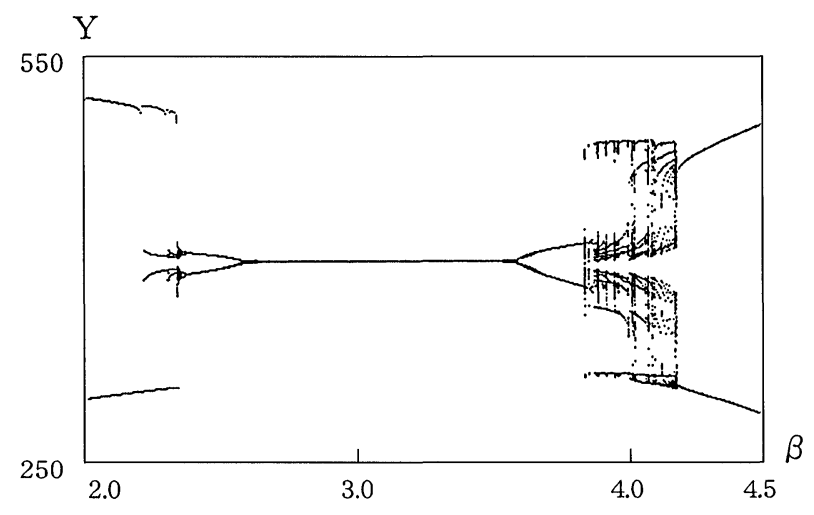

(a) $2.0 \leqq \beta \leqq 4.5$

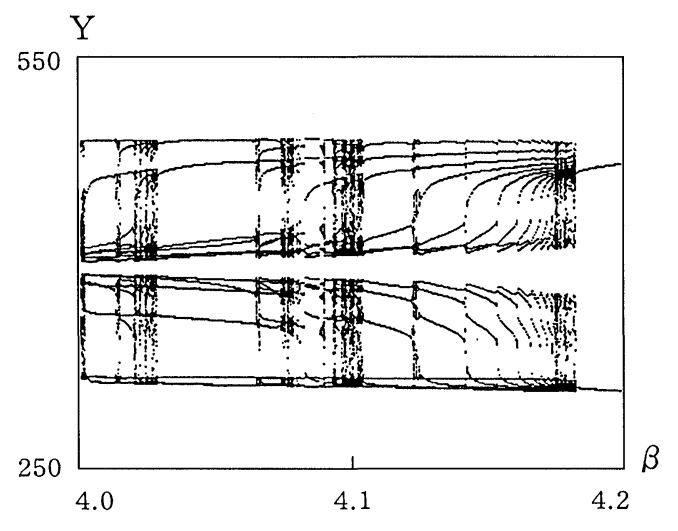

(b) $4.0 \leqq \beta \leqq 4.2$

FIGURE 12 Bifurcation diagrams of $Y$ when $\theta=0.3$ (parameter: $\beta$ ). 


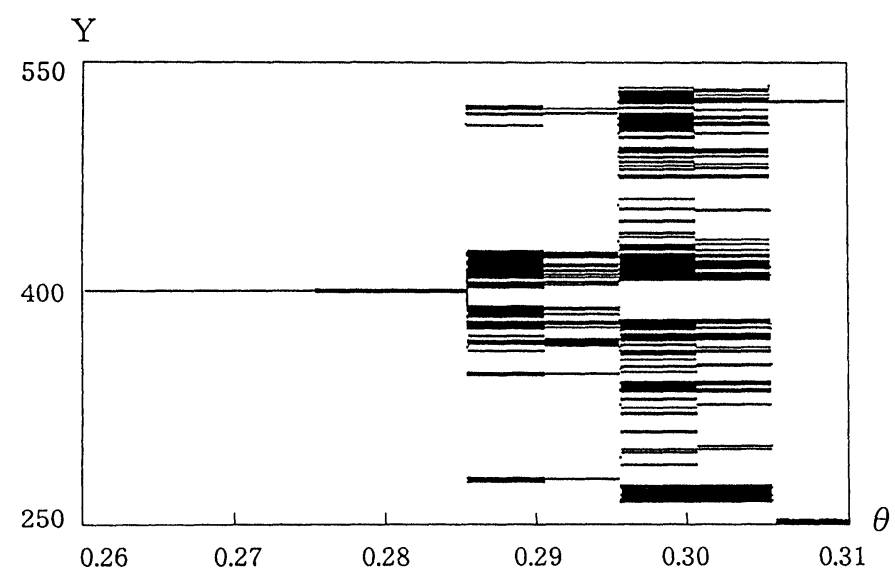

FIGURE 13 Bifurcation diagram of $Y$ when $\beta=4.1$ (parameter: $\theta$ ).

\section{CONCLUDING REMARKS}

In this paper, we formulated simple macrodynamic models with policy lag by means of mixed difference and differential equations, and investigated the effects of the policy lag on the dynamic behavior of the system analytically and numerically. We found that the too long lag must fail to stabilize the system, and in some situations cyclical movement occurs. Furthermore, we found that even the chaotic movement is possible for some parameter values in a model with variable capital stock. Nevertheless, it is not correct to say that the government's stabilization policy is entirely ineffective to stabilize the intrinsically unstable economy. In fact, the government can stabilize the economy when the policy lag is relatively short. In this sense, macroeconomic stabilization policy does not lose its significance even in the system with lags in policy response.

\section{Acknowledgements}

This is a slightly revised version of the paper which was presented at the Second International Conference on Discrete Chaotic Dynamics in Nature and Society (DCDNS2) which was held at Odense
University, Denmark (May 10, 2000). This research was financially supported by Chuo University, Nagoya Gakuin University, and Grant-inAid for Scientific Research No. 11630020 by Japan Society for the Promotion of Science.

\section{APPENDIX}

Proof of Proposition 2 Substituting $\rho=w \pm z i$ $(i \equiv \sqrt{-1})$ into the characteristic Eq. (16) in the text, we have

$$
(w-\alpha a)+\alpha \beta e^{-\theta w} e^{\mp \theta z i} \pm z i=0 .
$$

Rewriting Eq. (A1) by using the following 'Euler's formula'

$$
e^{ \pm i x}=\cos x \pm i \sin x
$$

we have the following expression.

$w-\alpha a+\alpha \beta e^{-\theta w} \cos \theta z+\left[ \pm z \mp \alpha \beta e^{-\theta w} \sin \theta z\right] i=0$.

From Eq. (A3) we obtain the following nonlinear system of equations with two unknowns, $w$ and $z$.

$$
\begin{gathered}
w=\alpha a-\alpha \beta e^{-\theta w} \cos \theta z \\
z=\alpha \beta e^{-\theta w} \sin \theta z
\end{gathered}
$$


We can solve this system of equations by adopting the method by Frisch and Holme (1935). ${ }^{17}$ We can rewrite Eq. (A4b) as

$$
e^{\theta w}=(\theta \alpha \beta \sin \theta z) / \theta z
$$

or equivalently,

$$
w=[\log \theta \alpha \beta+\log (\sin \theta z / \theta z)] / \theta .
$$

Substituting Eqs. (A4b) and (A6) into Eq. (A4a), we obtain the following equation with only unknown, $z$.

$$
\begin{aligned}
\xi(\theta z) & \equiv(\theta z / \tan \theta z)+\log (\sin \theta z / \theta z) \\
& =\theta \alpha a-\log \theta \alpha \beta \equiv E(\theta, \beta)
\end{aligned}
$$

Let us fix $\left(\theta_{0}, \beta_{0}\right)=\left(\theta_{0}, \psi\left(\theta_{0}\right)\right) \subset C$. In the region $C$ there is no real root, and in this region we have $\beta>\varphi(\theta)$, which implies that

$$
E\left(\theta_{0}, \beta_{0}\right) \equiv \theta_{0} \alpha a-\log \theta_{0} \alpha \beta_{0}<1 .
$$

In this case, we obtain Figure A1. This figure shows that there exist the solutions $z_{h}$ such that

$$
2 h \pi / \theta<z_{h}<(2 h+1) \pi / \theta
$$

for all $h \in\{0,1,2,3, \ldots\}$. In other words, there exist infinite number of solutions. Substituting $z_{h}$ into Eq. (A6), we have the solutions for w, i.e.,

$$
\begin{gathered}
w_{h}=\left\{\log \theta_{0} \alpha \beta+\log \left(\sin \theta_{0} z_{h} / \theta_{0} z_{h}\right)\right\} / \theta_{0}, \\
h \in\{0,1,2,3, \ldots\} .
\end{gathered}
$$

Eq. (A10) shows that $w_{h}$ is the increasing function of $\sin \theta_{0} z_{h} / \theta_{0} z_{h}$. This implies that

$$
w_{0}>w_{1}>w_{2}>w_{3}>\cdots
$$

in other words, the smallest imaginary part $z_{0}$ corresponds to the largest real part $w_{0}$ among the solutions (see Fig. A2).

It is clear that (when $\theta$ is fixed at $\theta_{0}$ ) at the values of $\beta$ which are slightly smaller than $\beta_{0}$, the system is locally stable so that the real parts of all roots are negative, and at the values of $\beta$ which are larger than $\beta_{0}$, the system is locally unstable so that the real part of at least one root is positive. This implies that $w_{0}=0$ and $d w_{0} / d \beta>0$ are satisfied at $\beta=\beta_{0}$, which means that the point

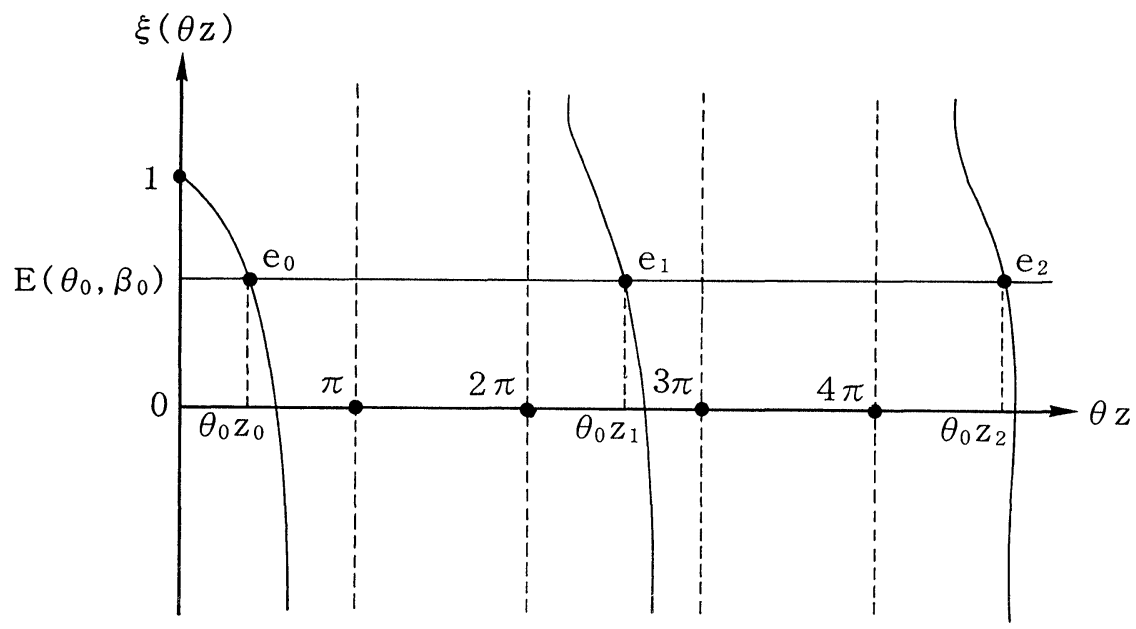

FIGURE A1 Solution of $z$.

\footnotetext{
${ }^{17}$ As for the method which is adopted here, see also Asada (1994).
} 


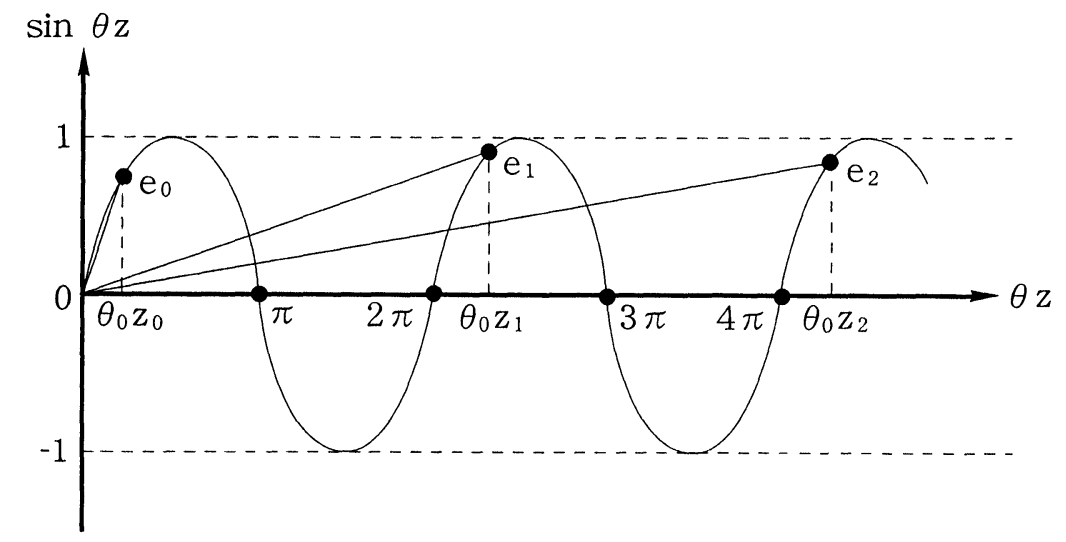

FIGURE A2 $\sin \theta_{0} z_{h} / \theta_{0} z_{h}(h=0,1,2, \ldots)$.

$\beta=\beta_{0}$ is in fact the Hopf-bifurcation point. Furthermore, from Eq. (A9) we have

$$
0<z_{0}<\pi / \theta_{0}
$$

so that the inequality

$$
2 \pi / \operatorname{Im} \rho\left(\beta_{0}\right)=2 \pi / z_{0}>2 \theta_{0}
$$

is satisfied.

Q.E.D.

\section{References}

Asada, T. (1991) "Lags in Policy Response and Macroeconomic Stability". The Economic Review of Komazawa University (Tokyo, Japan) 23-1, pp. 31-48.

Asada, T. (1994) "Monetary Factors and Gestation Lag in a Kaleckian Model of the Business Cycle". Semmler, W. (Ed.) Business Cycles: Theory and Empirical Methods, Boston: Kluwer Academic Publishers, pp. 289-310.

Asada, T. (1997) Macrodynamics of Growth and Cycles. Tokyo: Nihon Keizai Hyoron-sha. (in Japanese).

Asea, P. K. and Zak, P. J. (1999) "Time-to-Build and Cycles". Journal of Economic Dynamics and Control, 23, 1155-1175.

Bellman, R. and Cooke, K. L. (1963) Differential-Difference Equations. New York: Academic Press.

Friedman, M. (1948) "A Monetary and Fiscal Framework for Economic Stability". American Economic Review, 38, $245-264$.

Frisch, R. and Holme, H. (1935) "The Characteristic Solutions of a Mixed Difference and Differential Equation Occuring in Economic Dynamics". Econometrica, 3, 225-239.
Gandolfo, G. (1996) Economic Dynamics, Third Edition. Berlin Springer-Verlag.

Hayes, N. D. (1950) "Roots of the Transcendental Equation Associated with a Certain Difference-Differential Equation". Journal of London Mathematical Society, 25, 226-232.

Ioannides, Y. M. and Taub, B. (1992) "On Dynamics with Time-to-Build Investment Technology and Non-Time-Separable Leisure". Journal of Economic Dynamics and Control, 16, $225-241$.

James, R. W. and Belz, M. H. (1938) "The Significance of the Characteristic Solutions of Mixed Difference and Differential Equations". Econometrica, 6, 326-343.

Johansen, L. (1959) "Substitution versus Fixed Production Coefficients in the Theory of Economic Growth: A Synthesis". Econometrica, 27, 154-176.

Kaldor, N. (1940) "A Model of the Trade Cycle". Economic Journal, 50, $78-92$.

Kalecki, M. (1935) "A Macro-dynamic Theory of Business Cycles". Econometrica, 3, 327-344.

Kuang, Y. (1993) Delay Differential Equations with Applications in Population Dynamics. Boston: Academic Press.

Lange, O. (1969) Theory of Reproduction and Accumulation. Oxford: Pergamon Press.

Mackey, M. C. (1989) "Commodity Price Fluctuations: Price Dependent Delays and Nonlinearities as Explanatory Factors". Journal of Economic Theory, 48, 497-509.

Phillips, A. W. (1957) "Stabilization Policy and the TimeForm of Lagged Responses". Economic Journal, 67, 265-277.

Rustichini, A. (1989) "Hopf Bifurcation for Functional Differential Equations of Mixed Type". Journal of Dynamics and Differential Equations, 1(20), 145-177.

Steindl, J. (1952) Maturity and Stagnation in American Capitalism. Oxford: Oxford University Press.

Yoshida, H. (1999) "Fiscal Policy in Goodwin's Growth Cycle". Paper presented at 16th Pacific Regional Science Conference (PRSCO 16), July 1999, Seoul, Korea. 


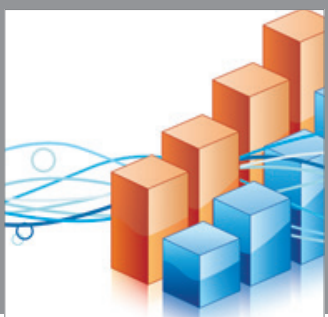

Advances in

Operations Research

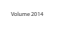

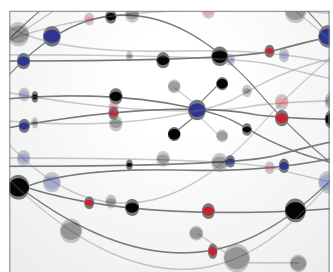

\section{The Scientific} World Journal
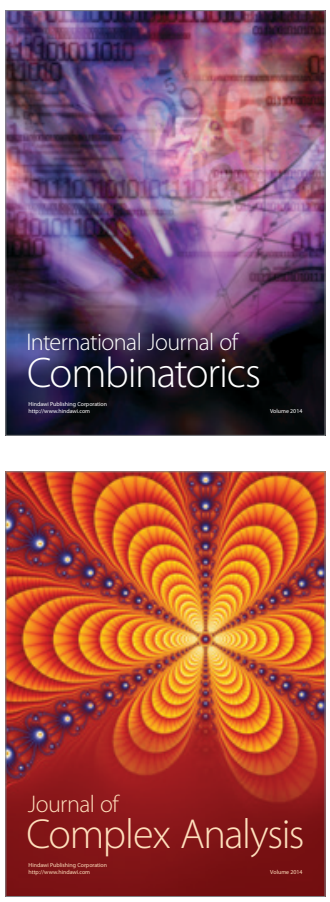

International Journal of

Mathematics and

Mathematical

Sciences
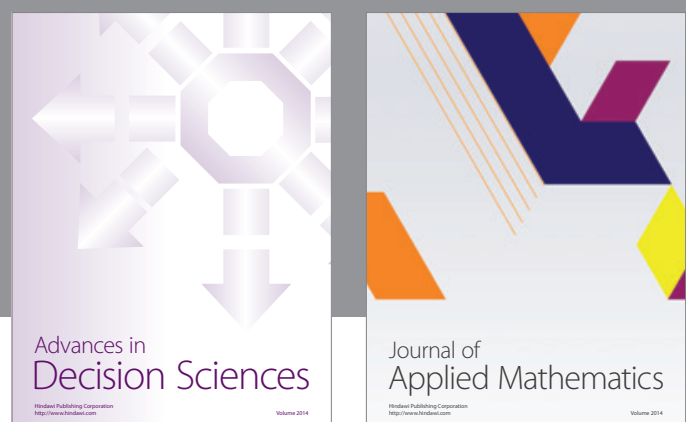

Journal of

Applied Mathematics
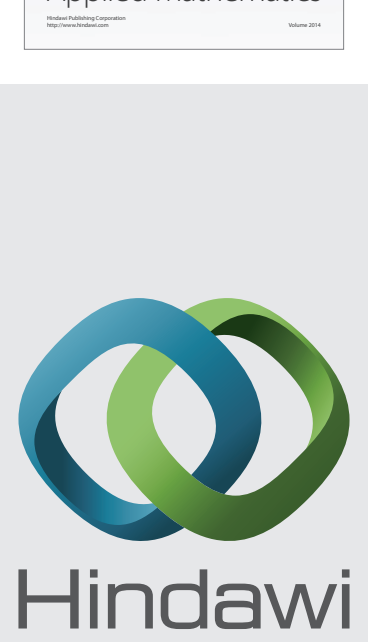

Submit your manuscripts at http://www.hindawi.com
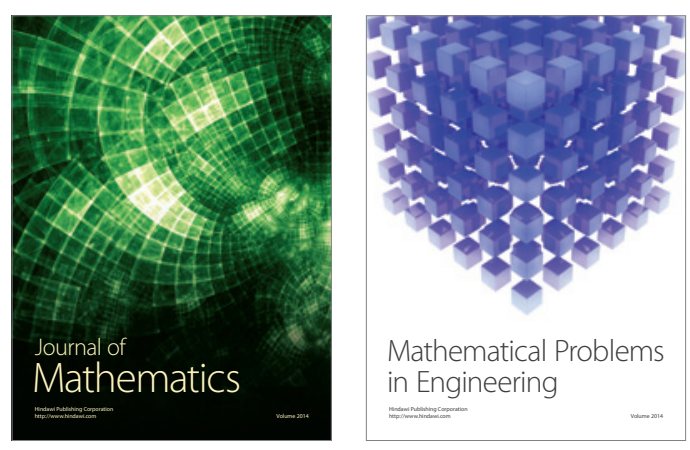

Mathematical Problems in Engineering
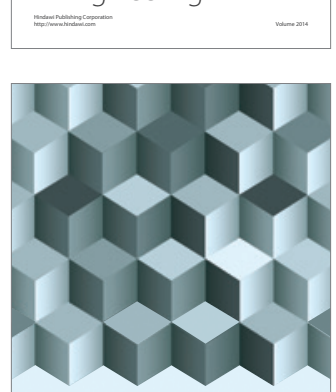

Journal of

Function Spaces
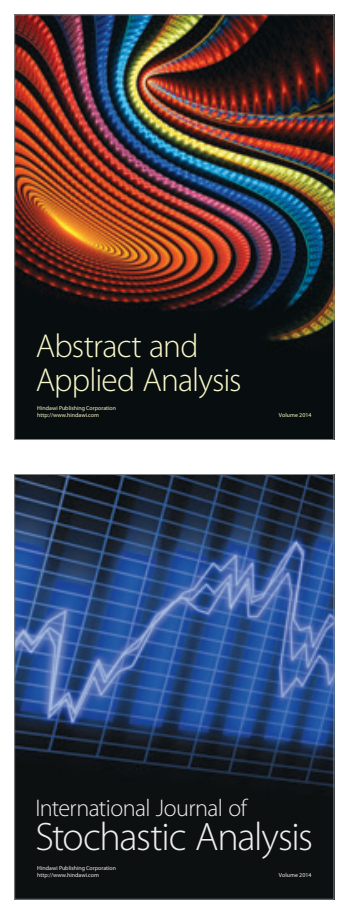

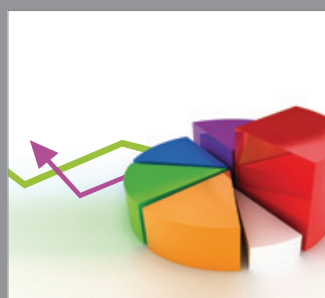

ournal of

Probability and Statistics

Promensencen
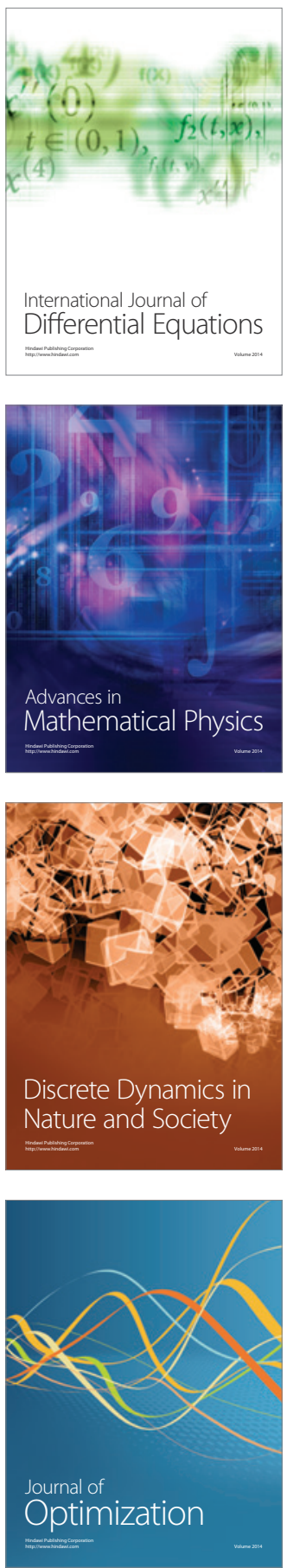